Quim. Nova, Vol. 35, No. 10, 2040-2043, 2012

\title{
ANÁLISE FORENSE: PESQUISA DE DROGAS VEGETAIS INTERFERENTES DE TESTES COLORIMÉTRICOS PARA IDENTIFICAÇÃO DOS CANABINOIDES DA MACONHA (Cannabis sativa L.)
}

\author{
Dayanne Cristiane Bordin e Marcos Messias \\ Faculdade de Ciências Farmacêuticas, Pontifícia Universidade Católica de Campinas, Av. John Boyd Dunlop, s/n, 13060-904 \\ Campinas - SP, Brasil \\ Rafael Lanaro \\ Laboratório de Toxicologia Analítica, Centro de Controle de Intoxicações, Universidade Estadual de Campinas, R. Carlos Chagas, \\ 150- $4^{\circ}$ andar, 13083-970 Campinas - SP, Brasil \\ Silvia Oliveira Santos Cazenave \\ Núcleo de Perícias Criminalísticas de Campinas, Instituto de Criminalística, Superintendência da Polícia Técnico Científica do \\ Estado de São Paulo, R. Barão de Parnaíba, 292, 13013-170 Campinas - SP, Brasil \\ José Luiz Costa* \\ Núcleo de Exames de Entorpecentes, Centro de Exames, Análises e Pesquisas, Instituto de Criminalística, Superintendência da \\ Polícia Técnico Científica do Estado de São Paulo, R. Moncorvo Filho, 410, 05507-060 São Paulo - SP, Brasil \\ Recebido em 19/1/12; aceito em 19/6/12; publicado na web em 18/9/12

\begin{abstract}
FORENSIC ANALYSIS: EVALUATION OF INTERFERING VEGETABLE DRUGS IN COLORIMETRIC TESTS FOR IDENTIFYING MARIJUANA CANNABINOIDS (Cannabis sativa L.).Marijuana (Cannabis sativa L.) is the most cultivated, trafficked and consumed illicit drug worldwide. Estimates indicate $10 \%$ of individuals experiencing marijuana become daily users, and 20-30\% use it weekly. Around 489 natural compounds have been identified in this plant, of which 70 are cannabinoids, responsible for psychic effects. The most relevant cannabinoid is 9-THC, recognized as the main chemical substance with psychoactive effects. The aims of this work was to investigate whether other drugs interfere with the colorimetric tests Fast Blue B and Duquenois-Levine, widely used for marijuana screening in forensic chemistry laboratories.
\end{abstract}

Keywords: marijuana; forensic chemistry; forensic toxicology.

\section{INTRODUÇÃO}

O consumo de substâncias psicoativas é uma característica comum à maioria das civilizações. De modo geral, essas substâncias foram e ainda são utilizadas em diversas épocas e culturas com finalidades terapêuticas, religiosas, lúdicas e para obtenção do prazer. A Cannabis sativa L., conhecida popularmente como maconha, é uma das plantas mais antigas que o homem tem conhecimento, com relatos de uso há mais de 4.000 anos. Originária da Ásia Central difundiu-se para o Brasil na época das capitanias, no final do século XVIII, destinada à produção de fibras; no entanto, acredita-se que a planta fosse conhecida há mais tempo e utilizada como hipnótico pelos escravos. ${ }^{1-3}$

É uma planta complexa que contém aproximadamente 480 substâncias químicas diferentes, distribuídas em 18 classes químicas. Dentre essas substâncias, destacam-se os óleos essenciais, flavonoides, açúcares, aminoácidos, ácidos graxos, compostos nitrogenados e terpenofenóis. A atividade farmacológica da planta está associada à classe terpenofenólica, composta por mais de 60 canabinoides, os quais não são encontrados em outras espécies vegetais. Eles são os responsáveis pelos efeitos da planta e classificados em dois grupos: os canabinoides psicoativos (por exemplo, $\Delta^{8}$-tetraidrocanabinol, (-)- $\Delta^{9}$-trans-tetraidrocanabinol $\left(\Delta^{9}-\mathrm{THC}\right)$ e o seu produto ativo, $\mathrm{o}$ 11-hidroxi-delta-9-tetraidrocanabinol) e os não psicoativos (por exemplo, canabidiol e canabinol). Dentre todos os canabinoides contidos na Cannabis sativa L., o $\Delta^{9}$-THC é, reconhecidamente, o principal composto químico devido ao seu pronunciado efeito psicoativo. É encontrado na planta madura, em concentração maior nas

*e-mail: jose.jlc@policiacientifica.sp.gov.br flores, com valores decrescentes nas folhas e somente em traços no caule e ramos; não é sendo encontrado nas raízes..$^{1,2,4-7}$ Sua estrutura química é mostrada na Figura 1.

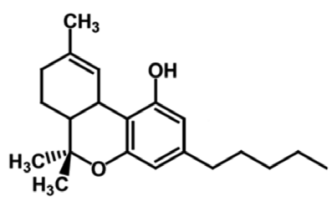

Figura 1. Estrutura química do (-)- $\Delta^{9}$-trans-tetraidrocanabinol $\left(\Delta^{9}-T H C\right)$

A maconha é a droga ilícita mais cultivada, traficada e consumida mundialmente. Dados estatísticos da Policia Federal dos últimos anos apontam que, no Brasil, a maconha é a droga com maior número de apreensões em todas as regiões do país, sendo que a principal região é a centro-oeste seguida pela sul, sudeste, norte e nordeste. Já nas estatísticas da Polícia Civil esse quadro de apreensões é equilibrado entre a maconha e a cocaína e seus derivados. ${ }^{8,9}$

De acordo com o relatório anual de 2008 da United Nations Office on Drugs and Crime (UNODC), cerca de 165,6 milhões de pessoas consumiram maconha entre 2006-2007. ${ }^{10}$ Estima-se que $10 \%$ dos que experimentaram se tornaram usuários diários e 20 a 30\% a consomem semanalmente. ${ }^{10}$

A lei federal $n^{\circ} 11.343$ de 23/08/2006, conhecida como Lei Antidrogas, em seu artigo 50 dispõe: "Para efeito da lavratura do auto de prisão em flagrante e estabelecimento da materialidade do delito, é suficiente o laudo de constatação da natureza e quantidade da droga, firmado por perito oficial ou, na falta deste, por pessoa idônea”. Assim, o laudo pericial é imprescindível para relatar as circunstâncias do fato, justificando as razões que levaram à classificação 
do delito, devendo indicar a quantidade e natureza da substância ou do produto apreendido, o local e as condições em que se desenvolveu a ação criminosa. ${ }^{11}$

As análises toxicológicas com finalidade forense podem fornecer evidências preciosas para materialização do crime e a base de um diagnóstico confiável é a realização de uma análise eficiente, sendo de fundamental importância o conhecimento da abrangência da técnica analítica empregada. Os métodos de triagem são empregados para verificar a presença ou ausência de uma determinada classe ou grupo de compostos. A escolha de um método de triagem é fundamental, pois define a gama de analitos que serão procurados e detectados. ${ }^{12,13}$

Os testes químicos mais utilizados para triagem da Cannabis sativa L. são Fast Blue B e Duquenóis-Levine. As reações colorimétricas que ocorrem nesses testes são atribuídas à natureza fenólica da estrutura química dos canabinoides e, por isso, falta-lhes especificidade, pois outros compostos análogos presentes nos vegetais podem se comportar de maneira semelhante. ${ }^{14,15}$ Assim, o presente trabalho teve como objetivo pesquisar se outras drogas vegetais interferem nestes testes químicos, amplamente utilizados em laboratórios de química e toxicologia forense.

\section{PARTE EXPERIMENTAL}

\section{Reagentes}

Reagente para teste de Fast Blue

Para preparo da solução dissolveram-se $0,025 \mathrm{~g}$ do sal Fast Blue B (cloreto de di-o-anisidina tetrazolio, Aldrich ${ }^{\circledR}$ ) em $10 \mathrm{~mL}$ de água destilada. A solução foi preparada no momento da análise.

\section{Reagente para teste de Duquenóis-Levine}

Para o teste de Duquenóis-Levine foi preparada a solução etanólica de vanilina $2 \%$. Pesou-se $1,0 \mathrm{~g}$ de vanilina $\left(\right.$ Aldrich $\left.^{\circledR}\right)$, adicionou-se etanol $96^{\circ} \mathrm{GL}\left(\right.$ Chemco $\left.^{\circledR}\right)$ até completa dissolução. Transferiu-se a solução para um balão volumétrico de $100 \mathrm{~mL}$, foram acrescentados $2 \mathrm{~mL}$ de ácido sulfúrico $\mathrm{R}\left(\right.$ Chemco $\left.^{\circledR}\right)$ e completou-se o volume com etanol $96^{\circ} \mathrm{GL}\left(\right.$ Chemco $\left.^{\circledR}\right)$.

\section{Amostras}

Foram selecionadas para as análises drogas vegetais com características macroscópicas semelhantes à Cannabis sativa. Analisaram-se 40 amostras de diferentes espécies de plantas disponíveis no estoque do laboratório de farmacognosia da Pontifícia Universidade Católica de Campinas (PUC-Campinas). Essas eram constituídas por todas as partes das plantas, moídas de forma homogênea, as quais se encontravam dentro do período de validade. A Tabela 1 apresenta os nomes popular e científico das drogas vegetais avaliadas como possíveis interferentes para o teste de triagem para maconha.

Para controle positivo dos testes colorimétricos foi utilizada amostra de maconha obtida junto ao Escritório das Nações Unidas sobre Drogas e Crime (UNODC), encaminhadas pelo programa International Collaborative Exercise - ICE de acreditação de laboratórios de toxicologia e química forense. Foram também utilizadas amostras de maconha de várias apreensões realizadas pelas forças policiais do estado de São Paulo e encaminhadas ao Núcleo de Perícias Criminalísticas de Campinas - Superintendência da Polícia Técnico Científica do Estado e São Paulo para análise. As amostras foram misturadas e submetidas à análise por cromatografia gasosa acoplada à espectrometria de massas para confirmação da presença dos principais canabinoides (canabinol, canabidiol, $\left.\Delta^{9}-\mathrm{THC}\right)$.
Tabela 1. Nome popular e científico das drogas vegetais submetidas aos testes colorimétricos Fast Blue B e Duquenóis-Levine

\begin{tabular}{|c|c|}
\hline Nome popular & Nome científico \\
\hline Arnica nacional & Solidago microgrossa $D C$ \\
\hline Arnica das montanhas & Arnica montana $L$. \\
\hline Beladona & Atropa belladonna $L$. \\
\hline Boldo do Chile & Peumus boldus Molina \\
\hline Calêndula & Calendula officinalis $L$. \\
\hline Camomila & Matricaria chamomilla $L$. \\
\hline Carobinha & Jacaranda decurrens Cham. \\
\hline Capim limão & Cymbopogon citratus (DC) Stapf. \\
\hline Cavalinha & Equisetum arvense $L$. \\
\hline Centella asiática & Centella asiática \\
\hline Chapéu de couro & $\begin{array}{l}\text { Echinodorus grandiflorus (Cham. \& Schltdl.) } \\
\text { Micheli. }\end{array}$ \\
\hline Confrei & Symphytum officinale $L$. \\
\hline Dedaleira & Digitalis purpurea $L$. \\
\hline Embaúba & Cecropia hololeuca Miq. \\
\hline Erva cidreira & Melissa officinalis \\
\hline Erva doce & Pimpinella anisum $L$. \\
\hline Erva de Santa Maria & Chenopodium ambrosioides $L$. \\
\hline Espirradeira & Nerium oleander $L$. \\
\hline Eucalipto & Eucalyptus globulus Labil. \\
\hline Folhas de mandioca & Manihot esculenta Crantz \\
\hline Fucus & Fucus vesiculosus $L$. \\
\hline Ginseng brasileiro & Pfaffia glomerata (Spreng.) Pedersen. \\
\hline Guaco & Mikania glomerata Spreng \\
\hline Guaraná & Paulinia cupana Kunth. \\
\hline Hamamélis & Hamamelis virginiana $L$. \\
\hline Hipérico & Hypericum perforatum $L$. \\
\hline Jaborandi & Pilocarpus jaborandi Holmes. \\
\hline Lobélia & Lobelia inflata $L$. \\
\hline Louro & Laurus nobilis $L$. \\
\hline Mamona & Ricinus communis $L$. \\
\hline Maracujá & Passiflora edulis $S$. \\
\hline Mate verde & Ilex paraguariensis St. Hill \\
\hline Menta & Mentha sp. \\
\hline Orégano & Origanum vulgare $L$. \\
\hline Pata de vaca & Bauhinia forficata Link. \\
\hline Picão preto & Bidens pilosa $L$. \\
\hline Poejo & Mentha pulegium $L$. \\
\hline Sálvia & Salvia officinalis \\
\hline Sene & Cassia angustifolia Vahl. \\
\hline Trombeteira & Datura suaveolens $L$. \\
\hline
\end{tabular}

\section{Métodos analíticos}

\section{Teste Fast Blue B}

Para realização do teste de Fast Blue B, uma alíquota de 100 mg de cada amostra de maconha ou droga vegetal foi transferida para 
frasco vidro incolor, com capacidade para $10 \mathrm{~mL}$, com tampa própria e submetida à extração com $2 \mathrm{~mL}$ de éter de petróleo. Cinco gotas do extrato etéreo (aproximadamente $250 \mu \mathrm{L}$ ) foram transferidas para papel de filtro e, após evaporação do solvente, foram adicionadas 5 gotas da solução do reativo de cor (Fast Blue B 0,25\%). O desenvolvimento de coloração vermelho-púrpura indica resultado positivo para o teste. ${ }^{15}$

\section{Teste Duquenóis-Levine}

Para o teste de Duquenóis-Levine, uma alíquota de 100 mg de cada amostra de maconha ou droga vegetal foi transferida para um frasco vidro incolor, com capacidade para $10 \mathrm{~mL}$, com tampa e submetida à extração com $2 \mathrm{~mL}$ da solução de vanilina etanólica $2 \%$. O extrato foi obtido através de filtração simples direta em tubo de ensaio. Em seguida, foram adicionados lentamente pelas paredes do tubo $2 \mathrm{~mL}$ de ácido clorídrico concentrado. A formação de anel azul-violáceo indica resultado positivo para o teste. ${ }^{15}$

\section{RESULTADOS E DISCUSSÃO}

Para controle de qualidade dos testes, as análises foram realizadas em triplicata. Foi utilizado também branco de reagentes, onde os testes foram realizados sem contato com nenhuma droga vegetal.

Nos controles positivos foram utilizadas misturas de amostras de Cannabis, obtidas de várias apreensões, com a finalidade de verificar a funcionalidade e a capacidade de detecção dos reagentes. A quantidade de substância ativa presente no vegetal é um importante interferente nos resultados e, portanto, deve-se levar em consideração a concentração mínima de ${ }^{9}-$ THC presente que esses testes sejam capazes de detectar. Plantas frescas, jovens, envelhecidas e frutos isolados podem gerar resultados duvidosos e até negativos nas análises preliminares. ${ }^{1,15}$

Além da análise química de triagem, é importante que a identificação da Cannabis seja complementada pelo exame dos caracteres macroscópicos da planta e, em seguida, seja analisada por exame microscópico. Os tricomas glandulares e tectores são os elementos microscópicos mais importantes na identificação da droga, o que nem sempre é fácil de visualizar pelo fato da planta se encontrar muito dividida e, por vezes, misturada com outros vegetais. Essas dificuldades aumentam quando a análise é destinada à identificação de haxixe, mistura resinosa obtida da maconha. ${ }^{1,15}$

Dentre as 40 drogas vegetais analisadas, no teste Fast Blue B foram observadas reações positivas, ou seja, o aparecimento instantâneo de uma coloração vermelho-púrpura semelhantes àquelas apresentadas por Cannabis sativa L., guaraná (Paulinia cupana Kunth.) e carobinha (Jacaranda decurrens Cham.). Nesse teste observou-se que a coloração dos extratos das plantas não influenciou os resultados.

A reação cromática de Fast Blue $\mathrm{B}$ foi atribuída à natureza fenólica da molécula dos canabinoides, o mecanismo reacional ocorre quando o extrato etéreo dos produtos da Cannabis sativa reage com o Fast Blue B, como mostra a Figura 2, formando um produto de cor vermelho-púrpura, que é solúvel na fase orgânica. A coloração formada é resultado da combinação de cores produzidas pela reação com diferentes canabinoides $(\mathrm{THC}=$ vermelho, canabinol = púrpura, canabidiol $=$ laranja) ${ }^{1}$

Para o teste de Duquenóis-Levine, dentre as 40 drogas vegetais

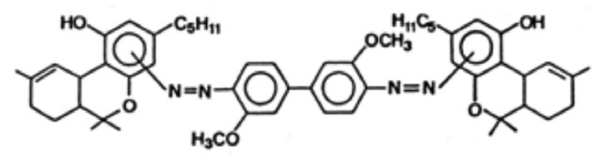

Figura 2. Possível produto de reação formado na reação de sal de Fast Blue analisadas, apresentaram resultados positivos as drogas vegetais boldo do Chile (Peumus boldus Molina), calêndula (Calendula officinalis L.), chapéu de couro (Echinodorus grandiflorus (Cham. \& Schltdl.) Micheli.), embaúba (Cecropia hololeuca Miq.), erva cidreira (Melissa officinalis), erva doce (Pimpinella anisum L.), guaraná (Paulinia cupana Kunth.), jaborandi (Pilocarpus jaborandi Holmes.) e louro (Laurus nobilis $L$.). Os resultados obtidos estão sumarizados na Tabela 2.

Tabela 2. Indicação das drogas vegetais que apresentaram resultados positivos no teste Fast Blue B (FB) e/ou Duquenóis-Levine (DL)

\begin{tabular}{lcclcc}
\hline Droga vegetal & FB & DL & Droga vegetal & FB & DL \\
\hline Boldo do Chile & - & + & Erva cidreira & - & + \\
Calêndula & - & + & Erva doce & - & + \\
Carobinha & + & - & Guaraná & + & + \\
Chapéu de couro & - & + & Jaborandi & - & + \\
Embaúba & - & + & Louro & - & + \\
\hline
\end{tabular}

$+=$ resultado positivo, $-=$ resultado negativo.

A reação de Duquenóis-Levine é apresentada na Figura 3. Os produtos do teste químico resultam na formação de uma cor vermelha, que se transforma em azul-violáceo quando se adiciona a amostra. O canabidiol, o ${ }^{9}$-THC e os ácidos destes compostos são responsáveis por essa reação. Com ácido clorídrico concentrado o íon carbânion terciário e, mais tarde, o íon carbânion ciclo hexenil são formados pelo deslocamento do íon hidreto, ocorrendo em equilíbrio com p-dimetilamino benzaldeído na presença de ácido clorídrico concentrado. A mudança de cor deve-se à desprotonação do grupo dimetilamino, como mostrado na Figura $3 .{ }^{15}$
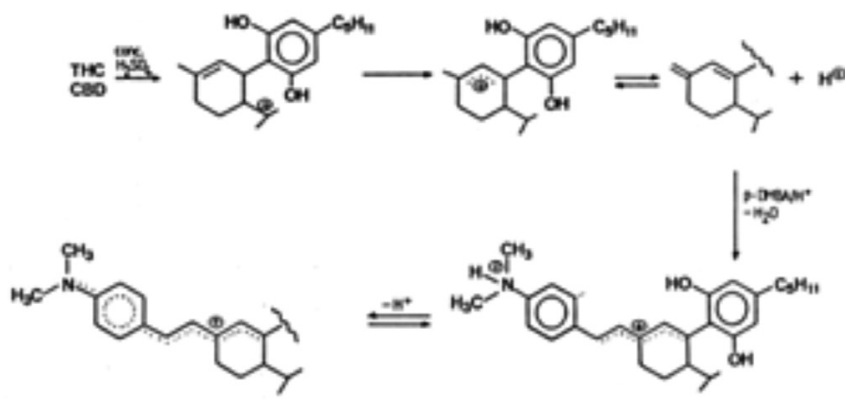

Figura 3. Reação de Duquenóis-Levine, entre os constituintes da Cannabis sativa e vanilina em meio ácido

Nesse teste notou-se que a cor do extrato pode influenciar na interpretação do resultado. Foi observado que nos extratos que apresentaram coloração intensa, como o jaborandi (verde-escuro) ou o boldo do chile, louro, calêndula e guaraná (coloração de vermelho intenso a vinho), a visualização do anel azul-violáceo pode ser dificultada. Deve-se considerar que a formação do anel azul-violáceo na Cannabis sativa L. é instantânea, já na análise de outras drogas vegetais o resultado por vezes se formava lentamente.

\section{CONCLUSÕES}

A partir dos resultados obtidos pode-se concluir que, quando comparado ao teste de Duquenóis-Levine, o teste Fast Blue B apresenta maior seletividade, uma vez que se obteve menor número de resultados falso-positivos para as drogas vegetais investigadas. A utilização concomitante dos dois testes preliminares pode aumentar a qualidade da análise de triagem. No entanto, é importante ressaltar que ambos devem ser realizados por profissionais capacitados e 
cientes das limitações do método.

Pelas limitações dos testes colorimétricos, é evidente que os resultados positivos obtidos nestes ensaios precisam obrigatoriamente ser confirmados por técnicas mais específicas, com princípios físico-químicos distintos do ensaio preliminar como, por exemplo, as técnicas cromatográficas e espectrométricas. Assim a qualidade final do laudo é garantida, evitando equívocos analíticos durante os procedimentos de instauração do inquérito policial.

\section{REFERÊNCIAS}

1. http://www.unodc.org/documents/scientific/ST-NAR-40-Ebook.pdf, acessada em Janeiro 2012.

2. McGuigan, M. Em Toxicologic Emergencies; Nelson, L. S.; Lewin, N. A.; Howland, M. A.; Hoffman, R. S.; Goldfrank, L. R.; Flomenbaum, N. E., eds.; McGraw-Hill: New York, 2006.

3. Ben Amar, M.; J. Ethnopharmacol. 2006, 105, 1.

4. Lanaro, R.; Dissertação de Mestrado, Universidade de São Paulo, Brasil, 2008.

5. Ribeiro, M.; Marques, A. C. P. R.; Laranjeira, R.; Alves, H. N. P.; de Araújo, M. R.; Baltieri, D. A.; Bernardo, W. M.; Lago, C.; Karniol, I.
G.; Kerr-Corrêa, F.; Nicastri, S.; Nobre, M. R. C.; de Oliveira, R. A.; Romano, M.; Seibel, S. D.; da Silva, C. J.; Rev. Assoc. Med. Bras. 2005, $51,247$.

6. Stambouli, H.; Bouri, A. E.; Bellimam, M. A.; Bouayoun, T.; Karni, N. E.; Bulletin on Narcotics 2005, 57, 79.

7. Wexler, P.; Encyclopedia of Toxicology, $2^{\text {nd }}$ ed.; Elsevier: Amsterdã, 2005.

8. http://www.dpf.gov.br, acessada em Outubro 2011.

9. Bressan, R. A.; Jungerman, F. S.; Laranjeira, R.; Rev. Bras. Psiquiatria 2005, 17, 5 .

10. http://www.unodc.org/documents/data-and-analysis/WDR2011/ World_Drug_Report_2011_ebook.pdf, acessada em Abril 2012.

11. http://www.planalto.gov.br/ccivil_03/_ato2004-2006/2006/lei/111343. htm, acessada em Janeiro 2012.

12. Chasin, A. A. M. Em Toxicologia Analítica; Moreau, R. L. M.; Siqueira, M. E. P. B., eds.; Guanabara Koogan: Rio de Janeiro, 2008.

13. Moreau, R. L. M. Em ref. 12.

14. Costa, A. F.; Farmacognosia, $5^{\text {a }}$ ed.; Fundação Calouste Gulbenkion: Lisboa, 2002.

15. http://www.unodc.org/pdf/scientific/SCITEC6.pdf, acessada em Janeiro 2012. 